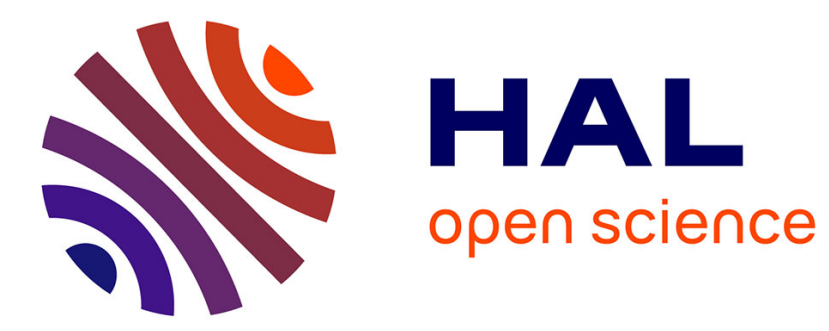

\title{
Comparing swimming performances of flexible and helical magnetic swimmers
}

\author{
Ali Oulmas, Johan E Quispe, Nicolas Andreff, Stéphane Régnier
}

\section{To cite this version:}

Ali Oulmas, Johan E Quispe, Nicolas Andreff, Stéphane Régnier. Comparing swimming performances of flexible and helical magnetic swimmers. 2019 IEEE/RSJ International Conference on Intelligent Robots and Systems (IROS), Nov 2019, Macau, France. pp.2415-2420, 10.1109/IROS40897.2019.8968485. hal-03391484

\section{HAL Id: hal-03391484 \\ https://hal.science/hal-03391484}

Submitted on 21 Oct 2021

HAL is a multi-disciplinary open access archive for the deposit and dissemination of scientific research documents, whether they are published or not. The documents may come from teaching and research institutions in France or abroad, or from public or private research centers.
L'archive ouverte pluridisciplinaire HAL, est destinée au dépôt et à la diffusion de documents scientifiques de niveau recherche, publiés ou non, émanant des établissements d'enseignement et de recherche français ou étrangers, des laboratoires publics ou privés. 


\title{
Comparing swimming performances of flexible and helical magnetic swimmers
}

\author{
Ali Oulmas ${ }^{1,2}$, Johan E. Quispe ${ }^{1}$, Nicolas Andreff ${ }^{2}$ and Stéphane Régnier ${ }^{1}$
}

\begin{abstract}
Flexible and helical magnetic microswimmers have been well reviewed in the literature because they could be exploited for envisaged applications such as targeted drug delivery, material removal, and micromanipulation. In this article, scaled-up versions of those robots are introduced to study in detail their maneuverability and dexterity while swimming. The robots were immersed in pure glycerol, thus, reproducing a low Reynolds scenario. The proposed robots were previously optimized, achieving their best performances. These magnetic swimmers have different actuation mode and geometric shape. The experiments assess the performances of these two kinds of robots in terms of rapidity, and steering error while following 3D trajectories in environments with high viscous variations.
\end{abstract}

\section{INTRODUCTION}

Nowadays, microrobots are good candidates to accomplish envisioned tasks such as minimally invasive diagnosis, targeted therapies, and cell/microobjects sorting [6]-[9], [14], [15], [21]. To achieve those tasks, a robot suitable to the environment anisotropy and the flow changes must be manufactured. In the biomedical context, these tasks or missions will be in biofluids through human conduits. Moreover, those fluids can pose additional constraints to the robot mission because of their fibrous networks [17]. Hence, it is preferred to look for a robot capable to overcome every issues presented. Notwithstanding, the above considerations, there exists biofluids such as the cerebrospinal fluid (CSF) that have a viscosity value closes to the water viscosity one [2] where could serve swimming techniques available for a large interval of low Reynolds number.

As consequence, this work aims at giving a comparison of the capabilities of the helical and flexible magnetic robots at milliscale by reproducing the low Reynolds condition using pure glycerol, and constrained the fabrication in such a way to obtain a free buoyancy millimeter robot. Both robots are tested in various settings considering their widespread control laws, namely giving a homogeneous rotating magnetic field for the helical swimmer, or an oscillating magnetic field for the flexible one. The settings are defined by different trajectories in different viscous environments, and the efficiency is measured in terms of rapidity to execute the trajectories, accuracy to accomplish properly the paths as

*This work was supported by EIPHI Graduate School (ANR-17-EURE0002), MultiFlag (ANR-16-CE33-0019-02) and Grand Prix Scientifique 2018 Ch. Defforey-Institut de France.

${ }^{1}$ A. Oulmas, J.E. Quispe and S. Régnier are with Sorbonne Université, CNRS, Institut des Systèmes Intelligents et de Robotique, ISIR, F-75005 Paris, France ali.oulmaseisir.upme.fr

${ }^{2} \mathrm{~N}$. Andreff and A. Oulmas are with Univ. Bourgogne FrancheComt/ CNRS, FEMTO-ST Institute F-25000, Besançon, France nicolas.andreffefemto-st.fr well as the manufacture constraints. These criteria are important for microobjects deliverance in order to accurately avoid obstacles and residues in those environments; Analysing the performance of the robot in viscosity changes can help to choose a swimmer which is able to work in a wide variety of (bio-) fluids with different viscosities; Finally, manufacturing process is determinant when it is required to change the swimmer features in order to optimize or adapt its shape for a specific task.

To enfranchise the challenges related to the microscale such as the microfabrication and the perception, a solution consists in studying macroswimmers at low Reynolds number configuration. To do so, it is possible to use a fluid with high viscosity such as glycerol or silicon oil. In this way, standard optical devices such as cameras can be used for 3D observation and tracking and the swimmers are easier to manufacture and customize. These swimmers serve as test benches before validating at microscopic scale. The experimental comparison results confirm the intuition that helical swimmers are more suitable to perform straight line trajectories than flexible ones. Conversely, the flexible swimmers are suitable to perform complex curves with wide range of fluid viscosities.

In the outline of this paper, section II presents the experimental set-up and the different magnetic actuation modes of the helical and flexible swimmers. In section II as well, kinematics equations involved in the system and the control law for the 3D motion control are briefly recalled. Sections III and IV are the core of this work. They define respectively the manufacturing and performances criteria and exhibit the results of helical and flexible swimmers comparison. Finally, section $\mathrm{V}$ gives conclusions and new perspectives for this work.

\section{EXPERIMENTAL SET-UP AND CONTROL}

The experimental system consists of a nested Helmholtz coils system capable of $10 \mathrm{mT}$ to actuate the swimmer robots. These robots are provided of neodymium permanent disc magnets in their heads (cf. Fig. 2). Following the procedure in [1], Navier-Stokes equation can be written in a dimensionless fashion in such a manner that the flow pattern only depends upon the Reynolds number. In [13], Purcell studied the limit condition when Reynolds number approaches zero. At this limit, inertial forces are negligible compared to viscosity forces, and microorganisms must perform a nonreciprocal pattern in order to swim. The authors in [5] reviewed this topic and gave an idea of the magnitude order of the Reynolds number of such microorganisms. For 

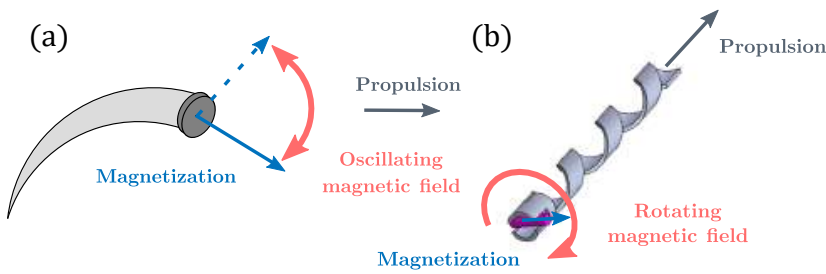

Fig. 1: Flexible and helical swimmer and their actuation mode by oscillating and rotation uniform magnetic fields in (a) and (b) respectively.

instance, in water, E. coli bacteria achieve a $\operatorname{Re}$ of about $10^{-5}$ to $10^{-4}$, a human spermatozoon a Re of approximately $10^{-2}$, the Paramecium a $\operatorname{Re}$ of around $10^{-1}$, and so on. In this article, the Reynolds number achieved by both robots is between $10^{-3}$ and $10^{-2}$. Since these millimetric robots are at low Reynolds, the comparison with their real counterparts at microscale is feasible.

With respect to the magnetic actuation, as magnetic field produced by the coils is practically uniform, the robots undergo only a magnetic torque $\mathbf{T}_{\mathrm{m}}$ as follows:

$$
\mathbf{T}_{\mathrm{m}}=\mathbf{M} \times \mathbf{B}
$$

where $\mathbf{M}$ is the magnetic moment and $\mathbf{B}$ the external magnetic field. The latter can be decomposed into driving and steering magnetic fields (respectively $\mathbf{B}_{\mathrm{d}}$ and $\mathbf{B}_{\mathrm{s}}$ ) as follows:

$$
\mathbf{B}=\mathbf{B}_{\mathrm{d}}+\mathbf{B}_{\mathrm{s}}
$$

The driving and steering magnetic field expressions will be detailed in the next sections for both swimmers. However, it is necessary to note that for characterization part only the driven magnetic field was necessary, being for helical propulsion $\left\|\mathbf{B}_{\mathrm{d}}\right\|=8 \mathrm{mT}$, and $\left\|\mathbf{B}_{\mathrm{d}}\right\|_{\text {max }}=10 \mathrm{mT}$ for flexible propulsion. But also can be written in this way, for helical robots $B_{0}=8 \mathrm{mT}$, and for flexible ones $B_{x}=2 \mathrm{mT}$ and $B_{y}$ $=8 \mathrm{mT}$, as will be seen.

\section{A. Helical swimmer magnetic actuation}

The helical swimmer is actuated by a rotating magnetic field as shown in Fig. 1. The driving and steering magnetic fields are respectively perpendicular and parallel to the principal axis of the robot. The magnetic torque tends to align the magnetic momentum of the swimmer with the applied magnetic field. Thus, with a rotating magnetic field $\mathbf{B}_{\mathrm{d}}$ and a helical tail, the swimmer propels itself converting its rotation into a linear movement thanks to the force generated by the interaction with the viscous fluid. The expression of the driving magnetic field $\mathbf{B}_{\mathrm{d}}$ for the helical robot is given as in [11] by:

$$
\mathbf{B}_{\mathrm{d}}=B_{0} \cos (2 \pi f t) \boldsymbol{y}_{\mathrm{B}}+B_{0} \sin (2 \pi f t) \boldsymbol{z}_{\mathrm{B}}
$$

where $f$ is the rotation frequency, $B_{0}$ is magnetic flux density in the center of the work-space while $\boldsymbol{y}_{\mathrm{B}}$ and $\boldsymbol{z}_{\mathrm{B}}$ are the base vectors of the plane perpendicular to the swimmer axis. The robot steering is made by $\mathbf{B}_{\mathrm{s}}$, which is defined by a proportional control of the geodesic error between the real axis of the swimmer and the desired axis [19]:

$$
\mathbf{B}_{\mathrm{s}}=-\operatorname{sign}\left(\mathbf{B}_{\mathrm{d}} \cdot \boldsymbol{x}_{\mathrm{B}}^{*}\right) \lambda\left\|\boldsymbol{x}_{\mathrm{B}} \times \boldsymbol{x}_{\mathrm{B}}^{*}\right\| \boldsymbol{x}_{\mathrm{B}}
$$

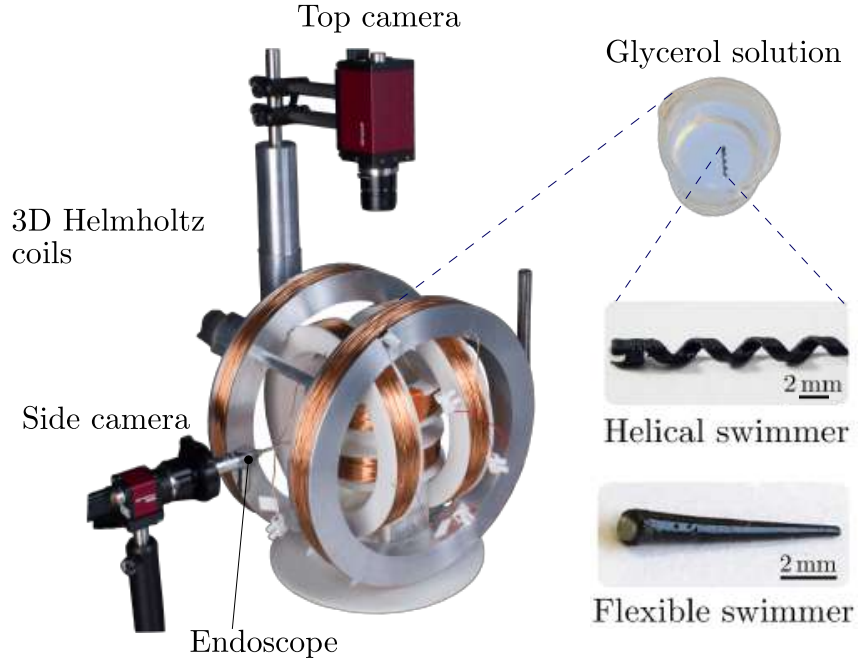

Fig. 2: The experimental set-up for the wireless magnetic manipulation of the helical and flexible swimmers at low Reynolds numbers.

where $\lambda$ is the proportional control gain and $\boldsymbol{x}_{\mathrm{B}}^{*}$ the desired orientation of the helical robot.

\section{B. Flagella motion-based actuation}

The flexible swimmer is actuated with an oscillating magnetic field. The tail twists when it follows the magnetic field and generates a propulsive force through the interaction with the viscous fluid. The propulsion principle is shown in Fig. 1. The driving magnetic field expression $\mathbf{B}_{\mathrm{d}}$ is given as in [3]:

$$
\mathbf{B}_{\mathrm{d}}=B_{\mathrm{x}} \boldsymbol{x}_{\mathrm{B}}+B_{\mathrm{y}} \cos (2 \pi f t) \boldsymbol{y}_{\mathrm{B}}
$$

where $\boldsymbol{x}_{\mathrm{B}}$ and $\boldsymbol{y}_{\mathrm{B}}$ define the swimming plane. Due to the fact that the linear movement direction is not changing with the oscillating magnetic field, a static and homogeneous magnetic field $\mathbf{B}_{\mathrm{x}}=B_{\mathrm{x}} \boldsymbol{x}_{\mathrm{B}}$ is added in order to control the flagella orientation. A sinusoidal magnetic field $\mathbf{B}_{\mathrm{y}}=B_{\mathrm{y}} \cos (2 \pi f t) \boldsymbol{y}_{\mathrm{B}}$ is applied in the direction perpendicular to the axis of the flexible robot, where $f$ is the oscillation frequency. The resulting magnetic field $\mathbf{B}_{\mathrm{d}}$ oscillates around the flexible swimmer axis. To achieve a desired orientation in space, the steering magnetic field $\mathbf{B}_{\mathrm{s}}$ is developed as follows:

$$
\mathbf{B}_{\mathrm{s}}=\frac{\lambda}{\|\mathbf{M}\|} \boldsymbol{x}_{\mathrm{B}}^{*}
$$

where $\lambda$ is the proportional control gain. The swimmers motion control in closed-loop is briefly introduced in II-C.

\section{Modeling and control}

The swimmer robots are modeled using a body-fixed frame $\mathcal{F}_{\mathrm{B}}=\left\{\begin{array}{lll}\boldsymbol{x}_{\mathrm{B}} & \boldsymbol{y}_{\mathrm{B}} & \boldsymbol{z}_{\mathrm{B}}\end{array}\right\}$ located at the robot center of mass $\boldsymbol{p}_{\mathrm{G}}$ with $\boldsymbol{x}_{\mathrm{B}}$ the principal axis of the robot. The orientation of the swimmer with respect to the global frame is characterized by the direction angle $\theta_{\mathrm{d}}$ (yaw) and the inclination angle $\theta_{\mathrm{i}}$ (pitch). The swimmer principal axis is related to these angles as follows:

$$
\boldsymbol{x}_{\mathrm{B}}=\left[\begin{array}{lll}
\mathrm{C} \theta_{\mathrm{i}} \mathrm{C} \theta_{\mathrm{d}} & \mathrm{C} \theta_{\mathrm{i}} \mathrm{S} \theta_{\mathrm{d}} & \mathrm{S} \theta_{\mathrm{i}}
\end{array}\right]
$$




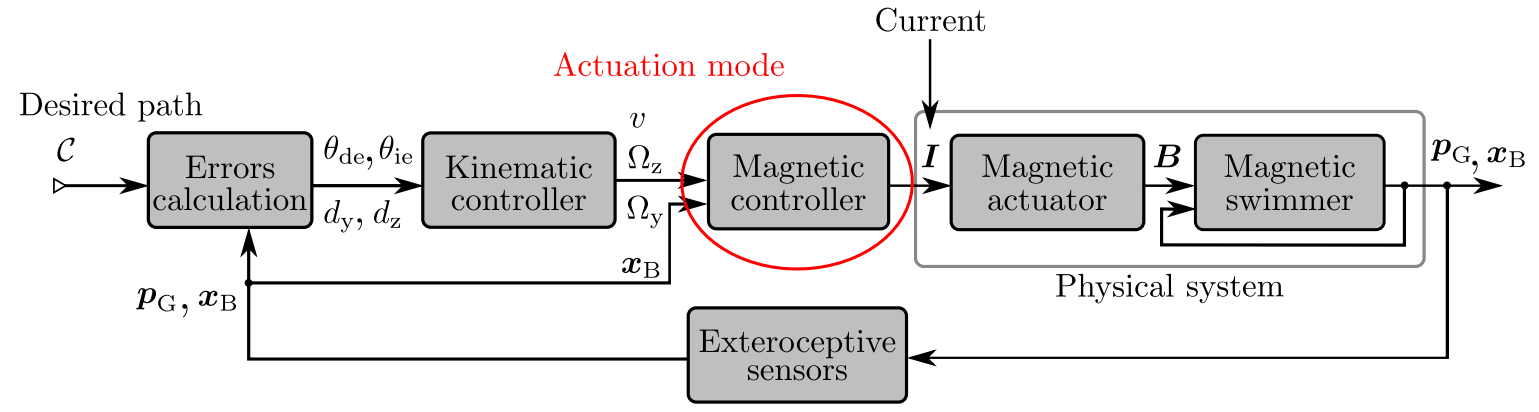

Fig. 3: Block diagram of the 3D path following for any magnetic swimmer robot behaving as a nonholonomic system. In order to change the propulsion mode specific to the swimmer, only the magnetic controller block is adapted.

This work uses an algorithm based on the path following problem, the aim is to minimize the distance and orientation errors between the swimmer robot and the reference path. Thereby, the state vector is defined as $\left(s, d_{\mathrm{y}}, d_{\mathrm{z}}, \theta_{\mathrm{de}}, \theta_{\mathrm{ie}}\right)$ where $s$ is the curvilinear abscissa of $p_{\mathrm{S}}$ (the orthogonal projection of the swimmer onto the path), $d_{\mathrm{y}}$ and $d_{\mathrm{z}}$ are the projection of the distance between the swimmer and the path on two axes perpendicular to the path tangent vector while $\theta_{\mathrm{de}}$ and $\theta_{\mathrm{ie}}$ are the two angles defining the orientation error between the swimmer velocity and the path tangent vector. The kinematics of the swimmers robot in the Serret-Frenet frame is given in [10]-[12]. To design the controller, an appropriate change of coordinates introduced in [10] allows us to transform the swimmer kinematic model into a 5states/3-inputs cascade system from which we were able to derive the following state feedback controller:

$$
\begin{aligned}
& u_{2}=-k_{\mathrm{d} 1} \dot{s} z_{2}-k_{\mathrm{t} 1}\|\dot{s}\| z_{3} \\
& u_{3}=-k_{\mathrm{d} 2} \dot{s} z_{4}-k_{\mathrm{t} 2}\|\dot{s}\| z_{5}
\end{aligned}
$$

where $k$. are positive control gains and $z$.. the state variables. The swimmers are actually driven by the steering angular velocity $\boldsymbol{\Omega}=\left(\begin{array}{lll}0 & \Omega_{\mathrm{y}} & \Omega_{\mathrm{z}}\end{array}\right)^{\mathrm{T}}$, which can be computed as a function of the control inputs:

$$
\begin{aligned}
& \Omega_{\mathrm{z}}=\gamma_{21}^{-1}\left(u_{2}-\gamma_{22}\right) \\
& \Omega_{\mathrm{y}}=\gamma_{31}^{-1}\left(u_{3}-\gamma_{33}-\gamma_{32} \Omega_{\mathrm{z}}\right)
\end{aligned}
$$

where $\gamma_{.}$. are appropriate scalars depending on the states and the path [10]. Finally, the desired orientation $\boldsymbol{x}_{\mathrm{B}}^{*}$ can be computed thanks to the time integration of the steering angular velocity:

$$
\boldsymbol{x}_{\mathrm{B}}^{*}=\boldsymbol{\Omega} \times \boldsymbol{x}_{\mathrm{B}} \mathrm{d} t
$$

where $\mathrm{d} t$ is the sampling time. To summarize, the block diagram of the entire algorithm, used to control the robot motions in space, is presented in Fig. 3. The path following algorithm (i.e. kinematic controller block on the figure) uses only the spatial errors between the robot and the reference trajectory, independently of the robot type. Thus, it is sufficient to adapt the actuation mode specific to the swimmer (i.e. the magnetic controller block) to switch from the helical swimmer motion control to the flexible one.

\section{COMPARISON OF TOPOLOGICALLY DIFFERENT SWIMMERS}

Helical and flexible swimmers use both non-reciprocal movements for displacing inside liquid media at low Reynolds number. However, they are completely different geometrically. Thereby, processes involved in the manufacturing are an important difference to consider. In this work, the helical swimmer has a rigid helical-shaped tail, fabricated using a 3D printer with "VisiJet M3 Black" material. Moreover, a disc-shaped magnet with $1.5 \mathrm{~mm}$ in diameter and $0.5 \mathrm{~mm}$ in height was attached to the swimmer head in such a way that the magnetization is perpendicular to the swimmer principal axis [11]. The second swimmer has a flexible tail, made from an elastomer (silicone) by mixing base and catalyst liquids, which cures at ambient temperature [12]. A certain ratio between the flexible swimmer elasticity and the fluid viscosity has to be respected. This relation is described by the sperm number:

$$
S_{\mathrm{p}}=\frac{\text { Viscosity forces }}{\text { Elastic forces }}
$$

For $S_{\mathrm{p}} \gg 1$, the tail is very flexible and its oscillations decrease rapidly because of the high viscosity value of the fluid. In the other case, $S_{\mathrm{p}}<<1$, the tail is rather rigid and it does not twist during the oscillations. In this situation, the swimmer movement is reciprocal and the "scallop theorem" is applied, thus, the swimmer velocity tends to zero. For the magnetization, the same disc-shaped magnet is attached to the flexible tail in such a manner to have the magnetization in the same direction as the robot principal axis. Some characteristics of the actual helical and flexible swimmers are summarized in TABLE I. Concerning the manufacturing, helical swimmers are straightforward to fabricate at millimeter scale rather than flexible swimmer because they can be manufactured using a 3D printer which allows to make complex-shaped and various swimmers. On the other hand, flexible swimmer requires to accomplish with the flexibility ratio or sperm number $(\mathrm{Sp})$ that means to take into account the material properties such as its Young modulus, its Poisson ratio, among others. In our particular case, we made use of a mold where base and catalyst are mixed strictly in 1:1 proportion in order to respect the ratio between the tail elasticity and the fluid viscosity. Besides, currently the 
TABLE I: Characteristics of helical and flexible swimmers.

\begin{tabular}{|l|c|c|}
\hline Characteristics & Helical swimmer & Flexible swimmer \\
\hline Length & $16 \mathrm{~mm}$ & $8 \mathrm{~mm}$ \\
\hline $\begin{array}{l}\text { Maximum } \\
\text { diameter }\end{array}$ & $1.5 \mathrm{~mm}$ & $1.5 \mathrm{~mm}$ \\
\hline Rigidity & $1.59 \mathrm{MPa}$ & $\approx 0.2 \mathrm{MPa}$ \\
\hline $\begin{array}{l}\text { Magnetization } \\
\text { direction }\end{array}$ & Radial & Axial \\
\hline Max. frequency & Cut-off & Optimal \\
\hline Speed variation & Rotation frequency & $\begin{array}{c}\text { Oscillation frequency } \\
\text { and amplitude }\end{array}$ \\
\hline
\end{tabular}

magnetization method (by surface functionalization or by providing a magnet) for both robots has not been completely mastered, especially at microscale. As the manufacturing of the helical swimmer is mastered at milliscale, we propose an experimental optimization of the helical swimmer geometry in III-A. Different swimmer shapes are proposed.

\section{A. Helical swimmer optimization}

Helical swimmers have been well reviewed in the literature [4]. Among the most relevant features, the axial pitch, length, number of turns and magnet shape are studied in various works [19]. However, the number of tails has not been considered yet in the case of rigid tail. In this subsection is presented an optimization with several helical tails considering a fixed length. In order to know how the number of tails affects the swimmer dynamics, different helical swimmers are designed (cf. Fig. 4) by varying the number of tails and mass. Fig. 4 shows that the forward speed of helical swimmers depends on the rotation frequency and the number of tails. It is well known that the helical robot reaches its maximal value of forward speed at the cut-off or stepout frequency [4], [18]. From our new results, under the conditions described in the caption of Fig. 4, we can observe that the swimmer speed decreases by increasing the number of flagella. This could be caused by the hydrodynamic interactions of the flow surrounding the different flagella when the space between them becomes smaller. Consequently, the total speed of the swimmer decreases. Thus, in this paper, helical swimmers with single flagellum present better performances in terms of propulsion and will be used in the rest of comparison experiments below. Conversely to the intuition, this result show that by increasing the number of flagella, speed decreases. Different to results demonstrated for multiple flexible flagella [20].

\section{COMPARISON OF SWIMMERS WITH DIFFERENT PROPULSION MODES}

In this work, two kinds of bio-inspired swimmers are designed, manufactured and their movements are controlled in closed-loop. However, which swimmer to choose to make specific trajectories in particular environments? This section tends to answer this question by comparing the swimmers experimentally using the curvature radii and the fluid viscosity.
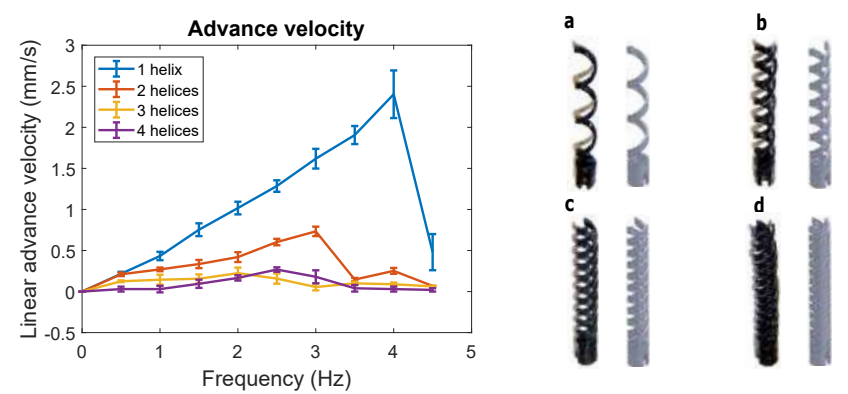

Fig. 4: On the right side, forward speeds vs rotation frequency in pure glycerol for the multi-tail helical-shaped robots. On the left side the manufactured robot and its respective CAD model for 1, 2, 3 and 4 tails in (a), (b), (c) and (d) respectively.

\section{A. Radius of curvature criterion}

The capacity of the swimmers to perform complex trajectories can be evaluated by studying their performances in terms of accuracy and execution rapidity to different curvature radii of the curves to be realized. In actual applications, this criterion can help to choose the swimmer which is able to perform complex paths in environments full of obstacles and residues (such as blood) by keeping high accuracy. In this work, the helical and flexible swimmers are nonholonomic, which means that they are limited for performing paths with complex curvatures. For that, a series of experiments with circular trajectories of different radii are performed to evaluate their abilities. The paths realized by the flexible swimmer are depicted in Fig. 5 with curvature radii ranging from $1 \mathrm{~mm}$ to $5 \mathrm{~mm}$ with a pitch of $1 \mathrm{~mm}$. For the helical swimmer, the curvature radii of the circles are varying from $2 \mathrm{~mm}$ to $10 \mathrm{~mm}$ with an axial pitch of $2 \mathrm{~mm}$ instead of $1 \mathrm{~mm}$ as for the flexible swimmer in order to maintain the same ratio length/radius for each swimmer. In fact, the helical swimmer length is twice as large as the flexible swimmer length. The paths performed by the helical robot are shown in Fig. 5.a. To study the precision of these two swimmers, the root mean square errors (RMS) of the lateral distance $d_{\mathrm{y}}$ during the path following of different circles are reported in Figure 5.d. For the flagella, the error is less than $500 \mu \mathrm{m}$ in all cases. For the helix, the error is $1 \mathrm{~mm}$ for a radius of curvature of $2 \mathrm{~mm}$ and for the other curvature radii, the accuracy is quite satisfactory. Thus, if we take the swimmers at the actual size, the results show that the flexible swimmer is much more precise than the helical one. The performances of these swimmers are also studied in terms of execution rapidity. TABLE II shows the time that the two robots take to perform a circle turn.

TABLE II: Time spent by the swimmers to complete a turn in seconds. The curvature radii are normalized by the swimmer length. Hel and Fla present respectively the abbreviations of the helical and flexible swimmers.

\begin{tabular}{|c|c|c|c|c|c|c|}
\hline \multicolumn{2}{|c|}{ Normalized radii } & $12.5 \%$ & $25 \%$ & $37.5 \%$ & $50 \%$ & $62.5 \%$ \\
\hline \multirow{2}{*}{ Time (s) } & Hel. & 54.01 & 78.15 & 104.73 & 135.06 & 148.46 \\
\cline { 2 - 7 } & Fla. & 34.1 & 85.51 & 132.12 & 141.23 & 203.71 \\
\hline
\end{tabular}


(a)

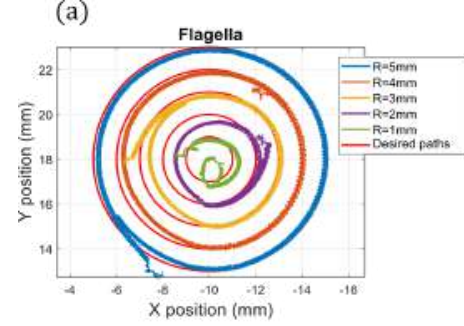

(b)

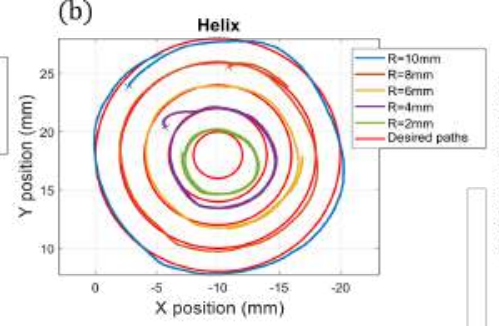

(c)

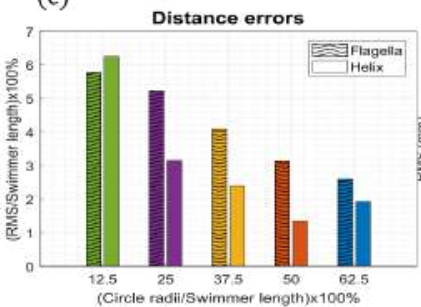

(d)



Fig. 5: Radii of curvature criterion. Circle tracking of the flexible (a) and helical (b) for different radii ranging from 1 to 5 mm for the flexible and from 2 to $10 \mathrm{~mm}$ for helical, constant gains were kept constant in both cases. (c) Lateral error graph normalized by the swimmer length. (d) Lateral distance RMS errors of the path following of the helical and the flexible.

It can be seen that except for a normalized radio of 12.5 , the helical swimmer is faster than the flexible one because it takes less time to make a turn of circle. To conclude, based on the experiments of this section, helical swimmers are more convenient to perform tasks with straight line trajectories because they are faster than the flexible ones but less accurate when the trajectory curvature becomes complex. On the other hand, flexible swimmers are more suitable for paths with complex curvature because they are accurate but very slow comparing to the helical swimmers. Furthermore, as the helical swimmer is twice as long as the flexible swimmer, a comparison can be made by normalizing the results and the curvature radii of the reference paths by the length of the swimmers, as illustrated in Fig. 5.c. The graph shows that for the same length, the precision performance of the helical swimmer could be better than the flexible one. To summarize, at the current state with the manufacturing processes that we master, the performance of the flexible swimmer is better than the helical robot in terms of accuracy during the path following of complex curvatures.

\section{B. Fluid viscosity criterion}

The swimmers dynamics depend strongly on the environment in which they are evolving, particularly the fluid viscosity. Their cut-off (or optimal) frequencies can change in function of the viscosity since the fluidic torque depends on it. To study the ability of the swimmers to operate in fluids with different viscosities, experiments have been performed using vertical sinusoidal trajectories in closedloop with two different viscosities of glycerol solution. First, a pure glycerol ( $99.99 \%$ with viscosity of $1.5 \mathrm{Pas}$ ) is used which is referenced as a high viscosity fluid for swimmers. Then, a $1 \%$ in volume of water is added, reducing the fluid viscosity to $20 \%$ from the initial value (from 1.5 to 1.2 Pas [16]). This is referred as a low viscosity fluid in these experiments. The rotation and oscillation frequencies of the magnetic field are kept fixed to the maximal values for all the experiments, as well as, the control gains.

For high viscosity, the results are depicted in Figure 6.a. It can be seen that both swimmers converge towards the reference path and follow well the trajectory. The distance quadratic errors of both swimmers once on the path $(\mathrm{X} \approx$ $23 \mathrm{~mm}$ ) are less than $200 \mu \mathrm{m}$. For comparison, the same experiments are performed with a lower fluid viscosity. In actual applications, the fluid is not always homogeneous.
Therefore, it is important to evaluate the behaviours of these swimmers in different conditions such as different fluid viscosities. The results of the path following are illustrated in Figure 6.b. It is noticed that the flexible swimmer converges towards the desired trajectory even in low fluid viscosity. However, it can be clearly seen that the helical swimmer has difficulties to reach the desired path. In these conditions, the vertical velocities for both swimmers are measured for different inclination angles and are shown in figure 6.c. We can see that for the flagella, the vertical velocity increases proportionally with the inclination angle. However, the helix vertical velocity reaches a plateau after $30^{\circ}$ at a weak value. This explains why the helical robot can not follow the desired trajectory. From these experiments, we can conclude that the flexible swimmer would be potentially able to work in wide range of fluids with different viscosities comparing to the helical swimmer. These results are valid for the robots used in this paper. After swimmers optimization, these results may be different, however, the fluid viscosity remains an interesting criterion of comparison between robots with different topologies. Fig. 7 gathers different criteria discussed in this paper for the flexible and helical swimmers comparison. These criteria are tested experimentally and help to choose the most efficient swimmer for a given trajectory and viscosity.

\section{CONCLUSION}

This paper proposes an experimental comparison between the bio-inspired helical and flexible swimmers on complex trajectories using multiple criteria such as the curvature radii of the desired trajectories and the fluid viscosity. The presented results allow to choose the most efficient swimmer for a given fluid viscosity and reference trajectory. Thus, helical swimmers are more suitable to perform straight line trajectories and the flexible ones for complex curves with wide range of fluid viscosities. This study attempts to propose a set of criteria for comparing swimmers that present different topologies and propulsion modes. The aim is to provide guidelines for users to choose the most efficient robot relative to a specific task in the future. As the fluid motion around the microswimmer is characterized only by the Reynolds number, the propulsion at the microscopic scale should be similar to that of the macroscopic swimmers. As a next step, microswimmers will be investigated to validate this work at low scales. For that, the macroswimmers will 

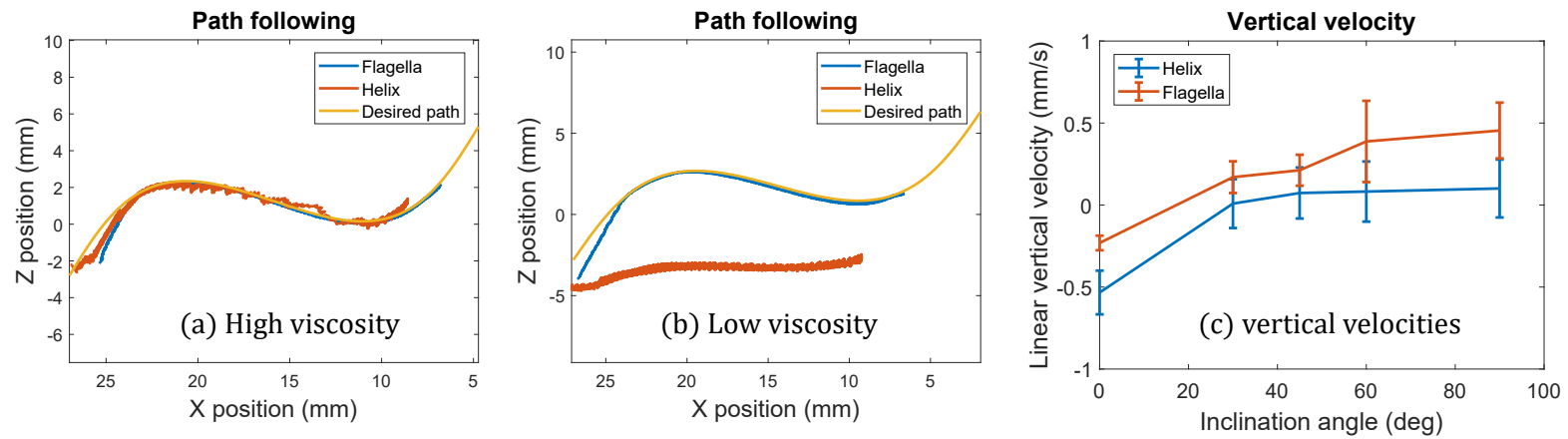

Fig. 6: Viscosity criterion. (a) Sinusoidal tracking trajectory of helical and flexible swimmer in the vertical plane ZX using a high viscosity fluid and results for low viscosity are depicted (b). (c) Depicts the vertical velocity of the helical and flexible swimmers using the low viscosity fluid.

be used as guidelines, for example, the ratios length/diameter and magnetic torque/fluidic torque will be kept the same to get the same performances. In addition, these comparison criteria will be used similarly to choose the most performant microswimmer as this study is dimensionless. As perspectives, it would be interesting to exploit more criteria such as boundary effects which can affect the swimmers motion. This measure can be important for tasks that take place in a confined space. The consumed energy criterion can be also studied.

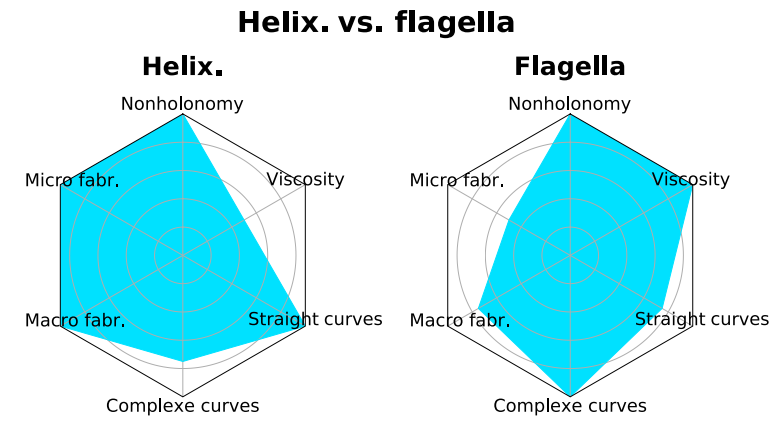

Fig. 7: Helical and flexible swimmers comparison with different criteria.

\section{REFERENCES}

[1] Jake J Abbott, Kathrin E Peyer, Marco Cosentino Lagomarsino, Li Zhang, Lixin Dong, Ioannis K Kaliakatsos, and Bradley J Nelson. How should microrobots swim? The international journal of Robotics Research, 28(11-12):1434-1447, 2009.

[2] IG Bloomfield, IH Johnston, and LE Bilston. Effects of proteins, blood cells and glucose on the viscosity of cerebrospinal fluid. Pediatric neurosurgery, 28(5):246-251, 1998.

[3] Rémi Dreyfus, Jean Baudry, Marcus L Roper, Marc Fermigier, Howard A Stone, and Jérôme Bibette. Microscopic artificial swimmers. Nature, 437(7060):862, 2005.

[4] Wei Gao, Xiaomiao Feng, Allen Pei, Christopher R Kane, Ryan Tam, Camille Hennessy, and Joseph Wang. Bioinspired helical microswimmers based on vascular plants. Nano letters, 14(1):305310, 2013.

[5] Eric Lauga and Thomas R Powers. The hydrodynamics of swimming microorganisms. Reports on Progress in Physics, 72(9):096601, 2009.

[6] Jinxing Li, B Esteban-Fernández de Ávila, Wei Gao, Liangfang Zhang, and Joseph Wang. Micro/nanorobots for biomedicine: Delivery, surgery, sensing, and detoxification. Sci. Robot., 2(4), 2017.

[7] Mariana Medina-Snchez, Lukas Schwarz, Anne K. Meyer, Franziska Hebenstreit, and Oliver G. Schmidt. Cellular Cargo Delivery: Toward Assisted Fertilization by Sperm-Carrying Micromotors. Nano Letters, 16(1):555-561, January 2016.
[8] Elvin M Muñoz, Johan E Quispe, and Emir Vela. Closed-loop selective manipulation of multiple microparticles by controlling the transient regime of marangoni flows. In 2016 IEEE/RSJ International Conference on Intelligent Robots and Systems (IROS), pages 51375142. IEEE, 2016.

[9] Bradley J Nelson, Ioannis K Kaliakatsos, and Jake J Abbott. Microrobots for minimally invasive medicine. Annual review of biomedical engineering, 12:55-85, 2010.

[10] Ali Oulmas, Nicolas Andreff, and Stéphane Régnier. Chained formulation of 3d path following for nonholonomic autonomous robots in a serret-frenet frame. In American Control Conference (ACC), 2016, pages 7275-7280. IEEE, 2016.

[11] Ali Oulmas, Nicolas Andreff, and Stéphane Régnier. Closed-loop 3d path following of scaled-up helical microswimmers. In Robotics and Automation (ICRA), 2016 IEEE International Conference on, pages 1725-1730. IEEE, 2016.

[12] Ali Oulmas, Nicolas Andreff, and Stéphane Régnier. 3d closed-loop motion control of swimmer with flexible flagella at low reynolds numbers. In 2017 IEEE/RSJ International Conference on Intelligent Robots and Systems (IROS), pages 1877-1882, Sept 2017.

[13] Edward M. Purcell. Life at low Reynolds number. American Journal of Physics, 45(1):3-11, January 1977.

[14] Johan Quispe, Elvin Muñoz, and Emir Vela. Exploiting the transient behavior of thermocapillary convection flows to enhance non-contact mesoscale manipulation. Applied Physics Letters, 109(12):124102, 2016.

[15] Johan E Quispe, Jean C Inga, Elvin M Muñoz, Stéphane Régnier, and Emir Vela. Single particle manipulation/sorting through the transient response of thermocapillary convection flows. In 2016 International Conference on Manipulation, Automation and Robotics at Small Scales (MARSS), pages 1-6. IEEE, 2016.

[16] Madison L. Sheely. Glycerol viscosity tables. Industrial chemistry, 24(9):1060-1064, 1932.

[17] Franziska Ullrich, Famin Qiu, Juho Pokki, Tianyun Huang, Salvador Pané, and Bradley J Nelson. Swimming characteristics of helical microrobots in fibrous environments. In Biomedical Robotics and Biomechatronics (BioRob), 2016 6th IEEE International Conference on, pages 470-475. IEEE, 2016.

[18] Tiantian Xu, Gilgueng Hwang, Nicolas Andreff, and Stéphane Régnier The rotational propulsion characteristics of scaled-up helical microswimmers with different heads and magnetic positioning. In Advanced Intelligent Mechatronics (AIM), 2013 IEEE/ASME International Conference on, pages 1114-1120. IEEE, 2013.

[19] Tiantian Xu, Gilgueng Hwang, Nicolas Andreff, and Stéphane Régnier. Characterization of three-dimensional steering for helical swimmers. In Robotics and Automation (ICRA), 2014 IEEE International Conference on, pages 4686-4691. IEEE, 2014.

[20] Zhou Ye, Stéphane Régnier, and Metin Sitti. Rotating magnetic miniature swimming robots with multiple flexible flagella. IEEE Transactions on Robotics, 30(1):3-13, 2013.

[21] Guanjia Zhao, Hong Wang, Samuel Sanchez, Oliver G Schmidt, and Martin Pumera. Artificial micro-cinderella based on self-propelled micromagnets for the active separation of paramagnetic particles. Chemical Communications, 49(45):5147-5149, 2013. 